\title{
Model predictive control of district heating system
}

\author{
Frode Lie-Jensen $^{1} \quad{\text { Andreas Aann } \emptyset^{1}}^{\text {Elena Aleksandrova }}{ }^{1} \quad$ Anders Westli $^{1} \quad$ Morten Nielsen $^{2}$ \\ Tiina Komulainen ${ }^{1}$ \\ ${ }^{1}$ Faculty of technology, Arts \& Design, Department of Mechanical, Electronic \& Chemical engineering, OsloMet, \\ Norway, timako@oslomet.no \\ ${ }^{2}$ Fortum Oslo Varme AS, morten.nielsen.luraas@fortum.no
}

\begin{abstract}
District heating system (DHS) is a widely used and increasingly popular energy source in cities. The uncertainty in the heat load (HL) due to customer demand fluctuations makes unit commitment (UC) and heat production unit (HPU) control a complex task. This case study of the DHS at Fortum Oslo Varme AS (FOV) aims to find a strategy to optimize and fully automate UC and HPU. Our results suggests this can be accomplished by using model predictive control (MPC) to control HPU power and flow rate, mixed integer linear programming (MILP) optimization to solve UC problem, and multiple linear regression (MLR) model to predict the HL. We also show that the fuel cost can be reduced significantly.

Keywords: district heating, model predictive control, system identification, unit commitment problem, heat load prediction
\end{abstract}

\begin{tabular}{cl}
\hline & \multicolumn{1}{c}{ NOMENCLATURE } \\
Symbol & Explanation \\
\hline & Plant variables \\
$K 1-4$ & HPU 1-4 \\
$T_{k t}$ & Temperature, outlet HPU \\
$T_{n t}$ & Temperature, outlet heat exchanger \\
$T_{k r}$ & Temperature, inlet HPU \\
$T_{n r}$ & Temperature, inlet heat exchanger \\
$\dot{V}$ & Volume flow, consumer side of heat ex- \\
& changer \\
$\dot{Q}$ & Power input to HPU \\
& Heat load prediction \\
$\alpha$ & constant \\
$y$ & predicted value, dependent variable \\
$x_{i}$ & independent variables \\
$\beta_{i}$ & regression constants \\
$\xi$ & error term \\
& Unit commitment optimization \\
$T_{h}$ & Time horizon \\
$\mathrm{N}$ & Number of HPU \\
$C_{n}^{t}$ & Energy cost for HPU $n$ at time $t$ \\
$P_{n}^{t}$ & Power production HPU $n$ at time $t$ \\
$P_{N}^{i}$ & Initial power HPU $n$ \\
$P_{e}^{t}$ & Predicted combined heat load
\end{tabular}

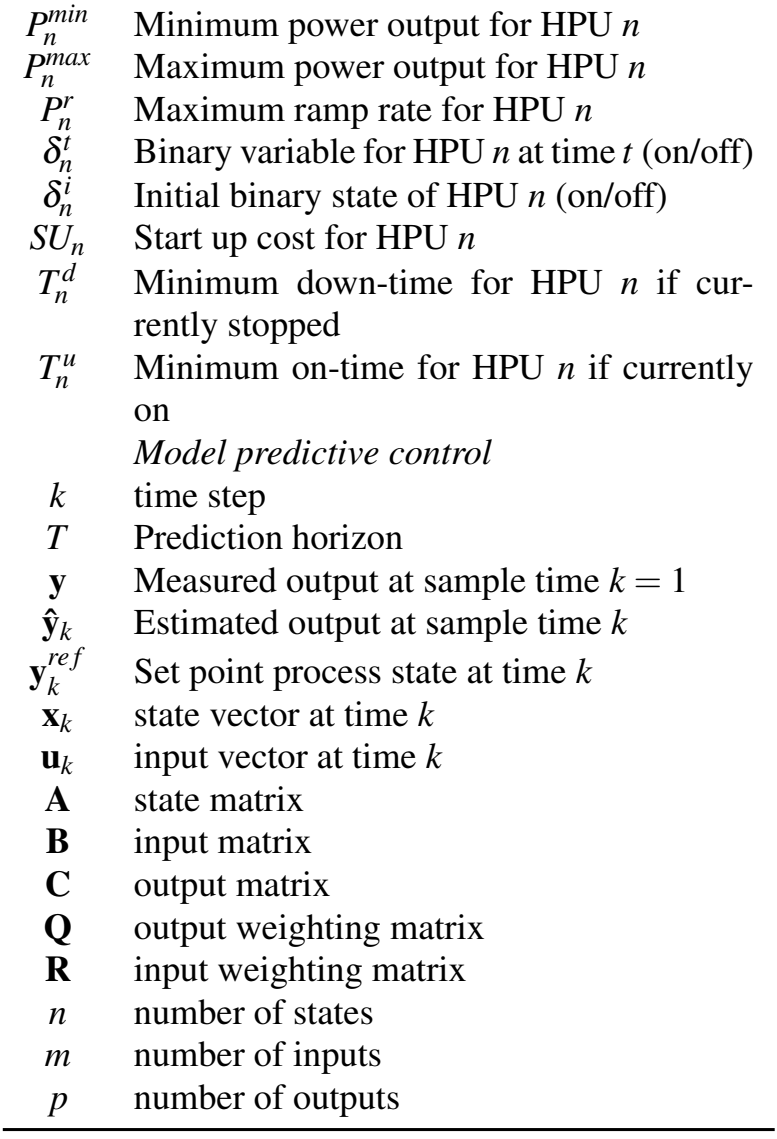

\section{Introduction}

District heating is a widely used technology for supplying hot water and space heating to consumers. The basic District Heating System (DHS) consists of Heat Production Units (HPU) applying energy to a closed system of circulating water. The energy is transported through the DHS to the customer substation that exchanges energy with the internal customer network, Figure 1. Early district heating networks were typically fueled by fossil fuels and supplied high temperature water or steam to the network. The last decades the focus has shifted towards renewable heat sources and maximizing operating efficiency (Lake, Rezaie, and Beyerlein 2017). In 2016 the distribution of energy sources for Norway was $50.1 \%$ waste incineration, $28.3 \%$ wood byproducts, $13.0 \%$ electricity, $5 \%$ fossil fuels and 3,6\% from other sources. (Statistisk Sentralbyrå 2017). 
Fortum Oslo Varme AS (FOV) owns and operates the DHS in Oslo and provides annually $1.7 \mathrm{TWh}$ to over 4000 consumers produced by 36 HPUs situated at 11 different Heat Production Plants (HPP). FOV provides $36 \%$ of the national combined power by DHS, making it the largest in Norway (Fortum Oslo Varme 2018).

FOV is continuously optimizing the DHS to minimize human resources, reduce fuel cost and improve operational reliability. One aspect that can contribute to this is to fully automate HPU commitment and control. FOV executes the HPU commitment problem and control manually by human resources, and due to the system complexity, the resulting commitment plan and HPU control is occasionally not optimal.

Considering the size of the FOV's DHS and number of HPUs, taking the entire system into account when solving these problems would be a large task. Therefore this paper aims to develop a strategy for automatic HPU control and commitment of a minor subsection of the DHS. The subsection selected for this project is separated from the rest of the DHS by a heat exchanger, and can easily be considered a separated system, Figure 1.

To optimize and automate HPU control and commitment, there are several aspect that need to be taken into consideration. The UC problem is dependent on knowing the future $\mathrm{HL}$, which is a variable that is customer controlled and fluctuates heavily depending on several factors such as season, temperature, wind, sunlight, time of day among others. A HL prediction is therefore necessary for achieving optimal UC results. Typically the UC problem in DHS operates with an hourly resolution and operators and simple controllers will handle any deviation from predicted value. The pitfall of this strategy is that it relies on operators executing the actual plan of the UC algorithm and making the right decision of which HPU to increase or decrease power. To eliminate this, a MPC can use the UC algorithm output to control all HPU on a shorter horizon based on the feedback from differential pressure at the substations.

\section{Heat load prediction}

In order to properly use predictive control and solving the UC problem, an estimate of future heat load is needed. Hagalid (2018) has as a part of his project thesis in collaboration with FOV developed a HL prediction based on multiple linear regression (MLR) for FOV's DHS. Other methods for predicting HL were not considered. A MLR model can be expressed as:

$$
y=\alpha+\sum_{k=1}^{n} \beta_{k} x_{k}
$$

Hagalid (2018) shows that, by selecting HL as dependent variable $(y)$ and air temperature, month of the year as 12 categorical variables, hour of the week as 168 categorical variables, heat production 24 hours prior, air temperature multiplied by month of the year, and air temperature multiplied by hour of the week as the independent variables $\left(x_{k}\right)$, results in robust and good performing model.

Hagalid kindly provided the MATLAB scrips used to create the MLR model. With minor adjustments the script was adapted to create a MLR model for the subsection. To find the the regression coefficients, the function fitlm was fed historical temperature and power data, The outdoor temperature is recorded at HPP 1 and power data is the hourly mean power transferred from HPP 1 and 2 to the subsection's substations.

\section{Unit commitment}

For district heating systems, involving more than one HPU, the state and how much power they should produce needs to be optimized. This will typically be influenced by variable fuel cost, restrictions on up/down-time, startup costs and ramp restrictions. Mixed integer linear programming (MILP) can be used to formulate a cost function to be minimized along with a number of constraints on the solution. Several strategies for solving this can be found in (Saravanan et al. 2013). Mixed integer problems are NP-hard and an efficient solver is therefore necessary.

Yalmip(Löfberg 2004) is a free MATLAB add-on which allows easier mathematical formulation of optimization problems. Optitoolbox (Currie and Wilson 2012) add-on provides an easier interface to MILP solver SCIP (Gleixner et al. 2017).

The cost function in (2) was implemented in MATLAB 2017b through the Yalmip/Optitoolbox add-ons using SCIP as solver. Constraints were applied to the solution in order to ensure correct initial conditions $(3,5)$, that power demand is met (6), power output is between HPU limits (7), ramp rate restrictions (8) and that up/down-time restrictions are met $(9,10)$.

$$
\min _{c} \quad J=\sum_{t=1}^{T_{h}} \sum_{n=1}^{N} C_{n}^{t} P_{n}^{t}+\sum_{t=2}^{T_{h}} \sum_{n=1}^{N} \delta_{n}^{t} S U_{n}
$$

$$
\begin{aligned}
& P_{n}^{i}=P_{n}^{t=1} \forall n \in N \\
& \delta_{n}^{t} \in\{0,1\} \forall n \in N, t \in T_{h} \\
& \delta_{n}^{i}=\delta_{n}^{t=1} \forall n \in N \\
& \sum_{n=1}^{N} P_{n}^{t} \geq P_{e}^{t} \forall t \in T_{h} \\
& \delta_{n}^{t} P_{n}^{\min } \leq \delta_{n}^{t} P_{n}^{t} \leq \delta_{n}^{t} P_{n}^{\max } \forall t \in T_{h}, \forall n \in N \\
& P_{n}^{r} \geq\left|P_{n}^{t}-P_{n}^{t-1}\right| \forall t \in T_{h}, \forall n \in N \\
& \delta_{n}^{u} \geq \delta_{n}^{t}-\delta_{n}^{t-1} \forall t \in T_{h}, \forall n \in N \\
& \delta_{n}^{u}=\left[\delta_{n}^{t}, \delta_{n}^{\min \left(T_{h}, t+T_{n}^{u}-1\right)}\right] \\
& \delta_{n}^{d} \leq 1-\delta_{n}^{t-1}-\delta_{n}^{t} \forall t \in T_{h}, \forall n \in N \\
& \delta_{n}^{d}=\left[\delta_{n}^{t}, \delta_{n}^{\min \left(T_{n}^{h}, t+T_{d}-1\right)}\right]
\end{aligned}
$$




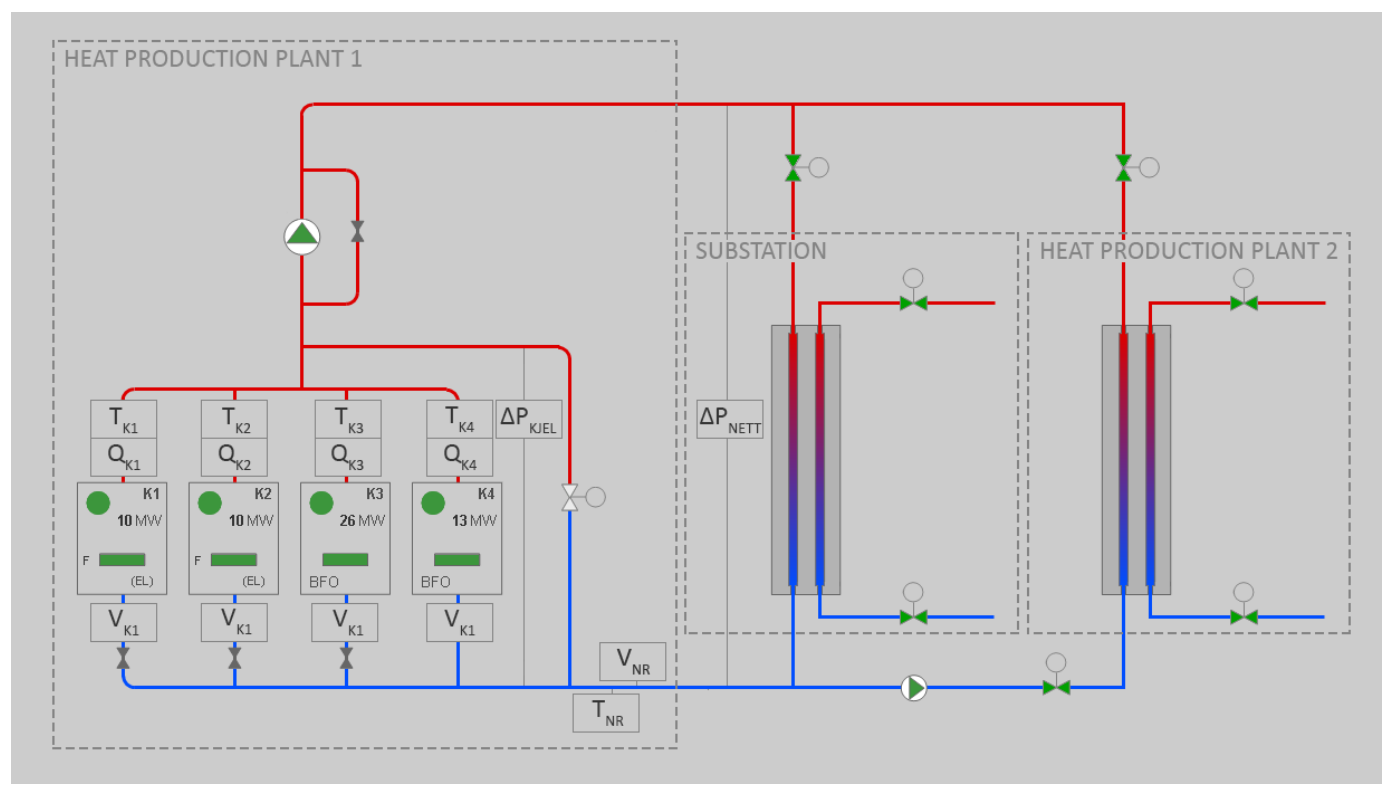

Figure 1. Operational sketch of the subsection of FOV's DHS. All substations are represented as one large. HPP 1, containing four HPUs(K1-K4), is the controlled plant. The subsection is separated from the rest of the DHS at HPP 2. Although not shown here, the HPUs have an internal loop that exchanges heat with the DHS

\section{System dynamics and identification}

The MPC is dependent on a model for predicting future system states. The models need to describe the dynamics of each HPU in the subsection. Although the HPUs use different fuel and are structurally diverse, they are all uniformly connected to the DHS, illustrated by Figure 2. This allows us to use the same model structure for all HPUs.

The variables of importance is the supply line temperature $\left(T_{n t}\right)$ and flow $\left(\dot{V}_{n}\right)$ to the DHS, hence selected as the model output. The flow in the internal loop is constant and is therefore omitted. The HPU outlet temperature $\left(T_{k t}\right)$ is not of interest of controlling to a given value. However MPC can provide predicted value for all outputs for the length of the simulation horizon, and $T_{k t}$ can be useful to calculate the HPU inlet temperature $\left(T_{k r}\right)$. Therefore $T_{k t}$ is also selected as model output. The variables affecting the selected outputs, hence selected as inputs, are the supplied HPU power $\left(\dot{Q}_{k}\right), T_{k r}$, the return line temperature from the DHS $\left(T_{n r}\right)$ and the reference flow value $\left(\dot{V}_{r e f}\right)$ for the DHS flow via the flow controlled valve.

There are multiple model types that fulfills our requirements. We have chosen to model our system as a process model transfer function (PMTF), defined as $H(s)=$ $\frac{k e^{-t s}}{\left(\tau_{1} s+1\right)\left(\tau_{2} s+1\right) \cdots\left(\tau_{n} s+1\right)}$ for $n$th order model with system gain $k$, time delay $t$ and time constants $\tau_{n}$. The resulting model structure for a HPU gives a multi-variable PMTF matrix is described in (11)

$$
\left[\begin{array}{c}
T_{k t}(s) \\
T_{n t}(s) \\
\dot{V}_{n}(s)
\end{array}\right]=\left[\begin{array}{cccc}
H_{11}(s) & H_{12}(s) & 0 & 0 \\
H_{21}(s) & H_{22}(s) & H_{23}(s) & H_{24}(s) \\
0 & 0 & 0 & H_{34}(s)
\end{array}\right]\left[\begin{array}{c}
\dot{Q}_{k}(s) \\
T_{k r}(s) \\
T_{n r}(s) \\
\dot{V}_{r e f}(s)
\end{array}\right]
$$

MATLAB System Identification Toolbox was used for model identification. Data with 2 second sample time was selected from the period September 2016 to April 2017. Emphasis was put on finding periods with varied operation where the HPU parameters showed sufficient dynamics. The HPUs have different dynamics during start-up and shut-down, due to by pass valves are open. These periods were avoided as estimation and validation data. Low order process models were sought, typically first order.

\section{Model predictive control}

MPC is a widely used concept. The basic principles involve calculating future response of outputs from a set of inputs based on a dynamic model of the plant. Both inputs and outputs are subject to constraints such as value and ramp rates. Measured or estimated disturbances can be incorporated in the system. MPC has the benefit of being able to keep inputs and outputs within specified operating ranges while keeping input variation to a minimum.

There are numerous ways to implement MPC, but it is most commonly applied as a linear quadratic cost optimization problem. Given a system described by the state space model $\mathbf{x}_{k+1}=\mathbf{A} \mathbf{x}_{k}+\mathbf{B} \mathbf{u}_{k}$, a quadratic cost optimization problem can be formulated as 


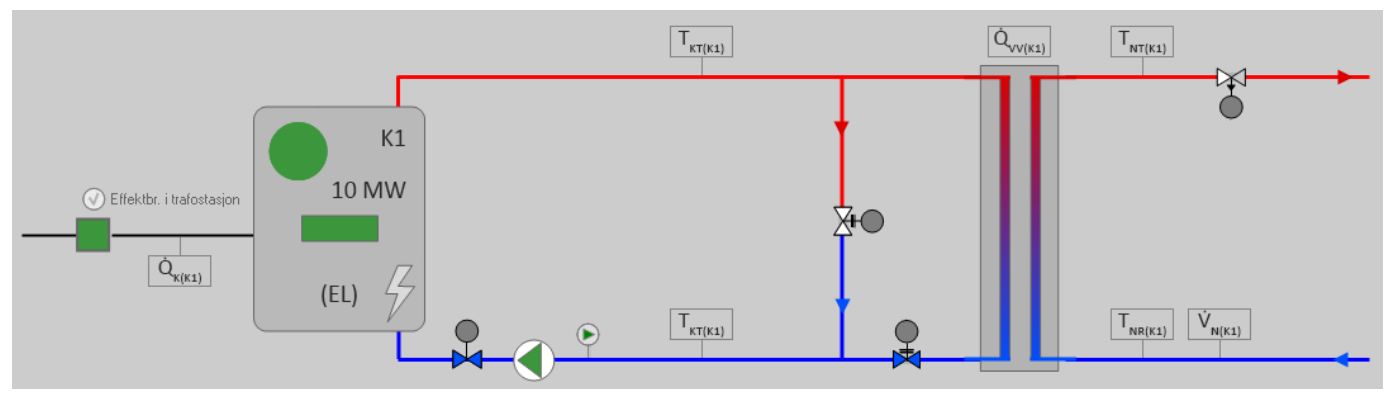

Figure 2. A operational sketch for HPU K1. The figure shows the internal loop of the HPU that exchanges heat to the DHS

$$
\begin{gathered}
\min _{\mathbf{u}, \Delta \mathbf{y}} J=\sum_{k=1}^{T_{h}} \Delta \mathbf{y}_{k}^{T} \mathbf{Q} \Delta \mathbf{y}_{k}+\mathbf{u}_{k}^{T} \mathbf{R} \mathbf{u}_{k} \\
\mathbf{x}_{k+1}=\mathbf{A} \mathbf{x}_{k}+\mathbf{B} \mathbf{u}_{k} \\
\hat{\mathbf{y}}_{k}=\mathbf{C} \mathbf{x}_{k} \\
\mathbf{x}_{\min } \leq \mathbf{x} \leq \mathbf{x}_{\max } \\
\mathbf{u}_{\min } \leq \mathbf{u} \leq \mathbf{u}_{\max } \\
\Delta \mathbf{u}_{\min } \leq \mathbf{u}_{k}-\mathbf{u}_{k-1} \leq \Delta \mathbf{u}_{\max } \\
\text { for } \quad \forall k \in\left[1, T_{h}\right] \\
\Delta \mathbf{y}_{k}=\mathbf{y}_{k}^{r e f}-\hat{\mathbf{y}}_{k} \\
\hat{\mathbf{y}}_{k=1}=\mathbf{y} \\
\mathbf{y} \in \mathbb{R}^{p}, \quad \mathbf{x} \in \mathbb{R}^{n} \\
\mathbf{u} \in \mathbb{R}^{m}, \mathbf{A} \in \mathbb{R}^{n \times n} \\
\mathbf{B} \in \mathbb{R}^{n \times m}, \quad \mathbf{C} \in \mathbb{R}^{p \times n}
\end{gathered}
$$

The model for one HPU consist of four inputs, however only $\dot{Q}_{k}$ and $\dot{V}_{\text {ref }}$ are controllable and set as manipulated variables (MV). $T_{k r}$ and $T_{n r}$ is set as measured disturbance (MD). A measured disturbance is often set to the present measured value for the whole simulation horizon. For $T_{n t}$ this is sufficient, as the temperature is stable with little variation. $T_{k r}$ on the other hand fluctuates heavily, which might reduce the MPC performance. To compensate for this, as mentioned in section 4 , future $T_{k r}$ values can be calculated using the predicted values $T_{k t}, T_{n t}$, the MD $T_{n r}$, the energy equation $\dot{Q}=c \rho \dot{V} \Delta T$ and the assumption $\dot{Q}_{\text {HEin }}=\dot{Q}_{\text {HEout }}$ as shown in (13)

$$
T_{k r}=-\frac{\dot{V}_{n}\left(T_{n t}-T_{n r}\right)}{\dot{V}_{i n t}}+T_{k t}
$$

The HPUs are parallel to each other, meaning the total flow $\dot{V}_{T}=\dot{V}_{K 1}+\dot{V}_{K 2}+\dot{V}_{K 3}+\dot{V}_{K 4}$. By combining the individual HPU flow outputs the resulting system model is a 16 by 9 PMTF matrix.

The MPC controller was made using MATLAB mpcfunction and simulated on the internal plant using $m p c$ move. Weights for the measured variables were tuned to allow considerable slack in $T_{k t}$ while keeping $T_{n t}$ and $\operatorname{dot} V$ as close to setpoint as possible. In order to handle the continuously fluctuating flow and at the same time foresee the largest time constants in the system, the sample time is set to 20s. With the simulation and control horizon set to respectively 100 and 50 samples, the MPC foresee 2000 seconds, about 33 minutes.

\section{System control strategy}

The simulation horizon of 33 minutes is sufficient to control the HPUs, but too short to make decision regarding $\mathrm{UC}$, hence a control hierarchy with differing time ranges is proposed. The prediction algorithm will run hourly and return predicted total power demand. The UC algorithm uses the predicted HL as input and returns operational state of each HPU to the model predictive controller. The UC algorithm can be run every 10-60 minutes or called when necessary from MPC. To integrate the UC and MPC algorithm, a function is needed, illustrated by Figure 3.

The function calculates $\operatorname{Re} f_{\dot{V}}$ for the length of the simulation horizon using $\dot{Q}_{\text {pred }}$ and $\dot{V}=\frac{\dot{Q}_{\text {pred }}}{c \rho\left(T_{n t}-T_{n r}\right)}$, knowing the present and predicted values of $T_{n t}$ and $T_{n r}$. The calculated $R e f_{\dot{V}}$ value might deviate from real time measurements of $\dot{V}$. The function creates a stipulated trajectory by regression towards $\dot{V}_{\text {ref }}$. The function also updates the MV cost and constraints to force the MPC to follow the UC algorithm plan.

The MPC is dependent on feedback to correct any deviation in predicted HL. There are no direct real time measurement of the HL available, but the differential pressure over the substations can work as a substitute. The customer controlled valve in the substations will open and increase the flow through the substation HE if the customer require more power. If all substations combined open their valves, and the total flow and temperature in the DHS are static, the differential pressure will drop. To compensate for this, one can either change the supply line temperature or the flow. Changing the supply line temperature implies large transport delays up to several hours, as the water flow average speed is $2-5 \mathrm{~m} / \mathrm{s}$. Changing the flow leads to a quicker response as the pressure wave in the pipes travels with speed up to $1200 \mathrm{~m} / \mathrm{s}$. To counter the deviations in the predicted HL, the total flow reference for HPP 1 can be adjusted by integrating the deviation from a set value for the substations differential pressure. The MPC will decide which of the enabled HPUs to increase power and flow. Consequently, the UC algorithm is not controlling the HPU power is directly, but decides which HPU to be active, start up or shut down. 


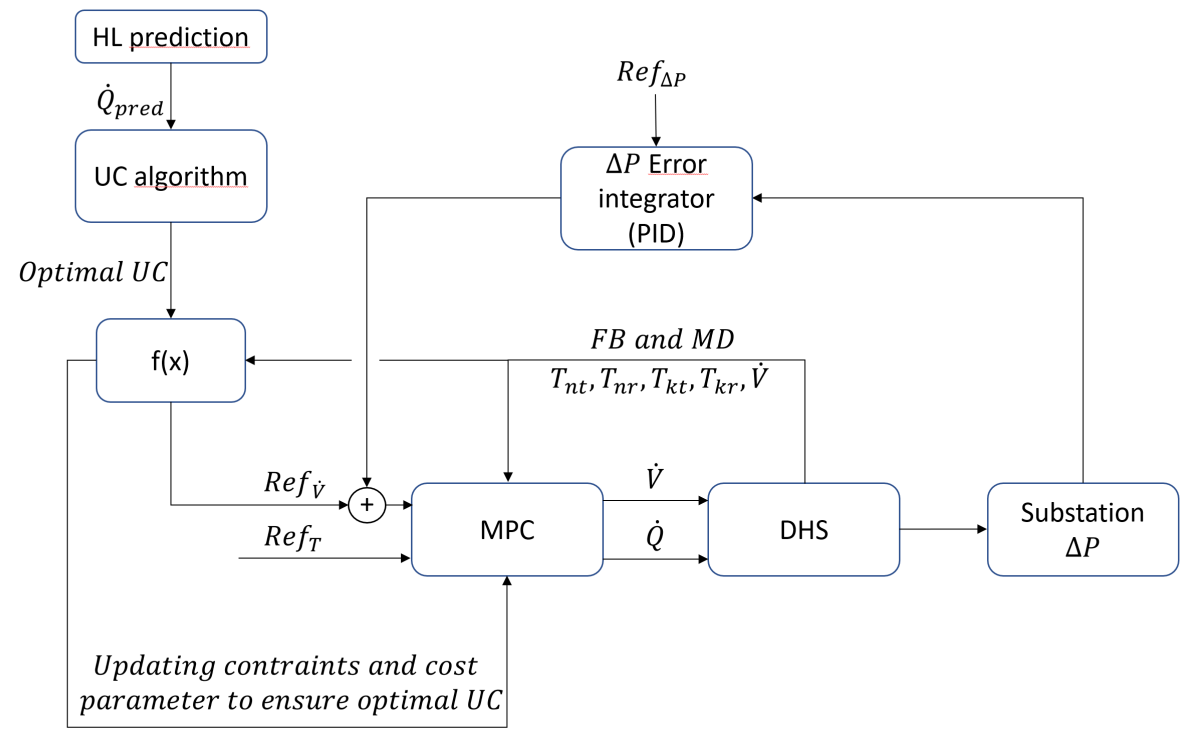

Figure 3. Figure show proposed system control hierarchy

\section{Results}

\subsection{Heat load prediction}

The HL predictions were made for three separate periods and compared to actual historical data. Plots of predicted values and actual data for the period 12-16. Jan 2016 is shown in Figure 4. Errors were calculated using Root Mean Square Error (RMSE), Mean Average Percent Error (MAPE) and Mean Error (ME), shown in Table 1.

From the error data from June 2017-February 2018 the mean error is negligible, this indicates that on average the model performs well. For shorter periods(16-20 Jan 2018) a significant bias is seen which might impair predictive control of the network. The MAPE and RMSE values are quite high but it is important to note that very short term deviations without bias can be tolerated in a district heating system. The short time deviations might not be model errors, but actually caused by variations in the operating mode of the HPUs so that the training data is flawed. A better model could probably be made by using consumer energy use instead of produced energy as inputs to the model.

Table 1. Errors for heat load prediction

\begin{tabular}{lllc}
\hline & $R M S E$ & $M A P E$ & $M E$ \\
Period & {$[M W]$} & {$[\%]$} & {$[M W]$} \\
\hline Jun 17-Feb 18 & 1.5 & 7.1 & 0.04 \\
12-16 Jul 17 & 0.8 & 9.5 & -0.09 \\
16-20 Jan 18 & 1.7 & 4.9 & -0.8 \\
\hline
\end{tabular}

\subsection{Unit commitment}

The unit commitment algorithm was tested against actual data from 20-24 Jan 2016 using the method described in section 3. Real, time dependent fuel prices were used.
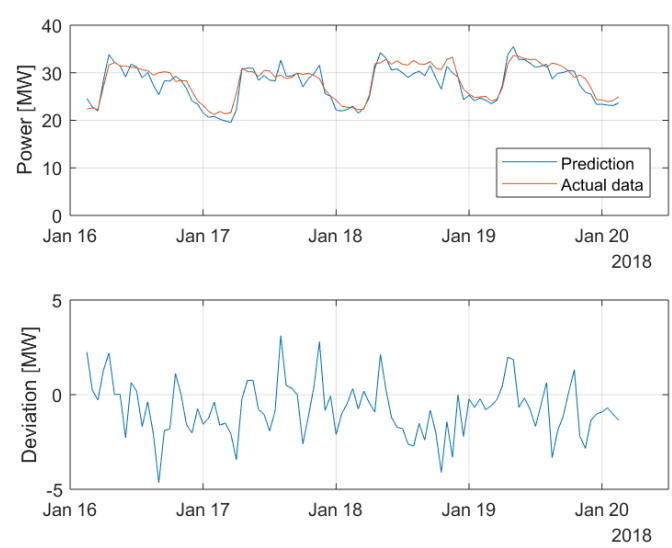

Figure 4. Predicted and actual power output for the period between 16-20 January 2018. Absolute deviation from actual data is shown in the lower part of the plot.

Since no data for consumer power usage were available power demand was calculated by summing the power output of all HPUs from historical data. The algorithm was also tested with the same numerical results, but slightly slower processing time using CBC (Lougee-Heimer 2003) and MATLABs intlinprog-solver.

Power output of each HPU from the algorithm and actual historical data are shown in Figure 5. Fuel prices are shown in the same figure for reference.

Fuel costs were calculated for actual data and the proposed solution. A potential fuel cost saving of $20.5 \%$ was seen for this period. However this was a known problematic period, similar savings are not to be expected routinely. The UC algorithm is powerful and returns good results, however this subsection of the district heating network is quite simple; only two types of HPUs of which none have difficult operating constraints nor any signifi- 
Table 2. Parameters for unit commitment optmization

\begin{tabular}{lcccc}
\hline$H P U(n)$ & $K 1$ & $K 2$ & $K 3$ & $K 4$ \\
\hline$P_{n}^{\max }[\mathrm{MW}]$ & 10 & 10 & 26 & 13 \\
$P_{n}^{\min }[\mathrm{MW}]$ & 0.5 & 0.5 & 0.5 & 0.5 \\
$P_{n}^{r}[\mathrm{MW} / \mathrm{hr}]$ & 48 & 48 & 60 & 60 \\
$T_{n}^{u}[\mathrm{hr}]$ & 1 & 1 & 1 & 1 \\
$T_{n}^{d}[\mathrm{hr}]$ & 1 & 1 & 1 & 1 \\
$\mathrm{SU}[\mathrm{NOK}]$ & 200 & 200 & 500 & 500 \\
\hline
\end{tabular}

cant start up costs. It might seem that the UC algorithm is overcomplicated for this use, but is chosen keeping in mind that it can be extended to handle UC for the entire DHS.

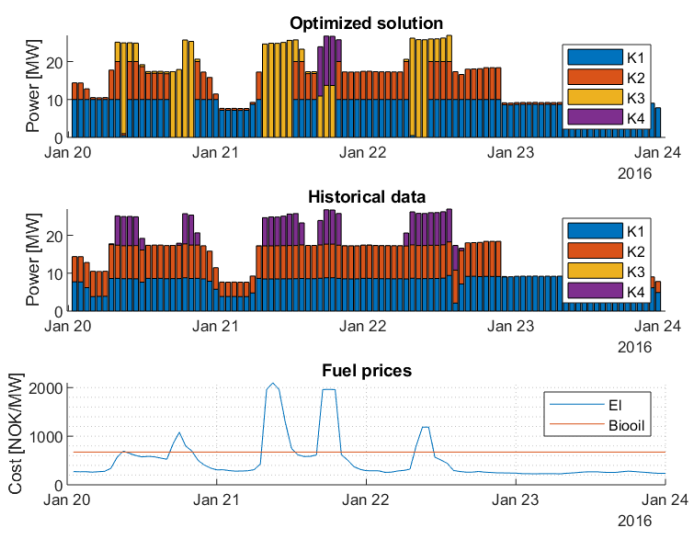

Figure 5. Top: Proposed solution from algorithm for the period 20-24 Jan 2016. Mid: Actual power output from historical data for the same period. Bottom: Actual fuel prices from the same period.

\subsection{System identification}

All four HPU loops were modeled separately as described in section 4, for brevity complete data will just be presented for HPU K1. Data for estimation was collected from the period 8-11 Feb 2017 and the resulting model was validated against data from 12-16 Jan 2016. The Normalised Root Mean Square Error (NRMSE) of 0.932 for $T_{k t}$ and 0.787 for $T_{n t}$ indicates a good fit between identified model and actual HPU dynamics. Plots of simulated response from model and validation data are shown in Figure 6 and 7 for respectively $T_{k t}$ and $T_{n t}$.

All models were process models as shown in (11), maximum model order was set to 2. Parametrization of all non-zero transfer functions for HPU 1 is shown in Table 3.

Table 4 shows a summary of NRMSE fits from system identification of all four HPUs. The electrical HPU (K12) yielded a good fit between model and actual data but larger deviations was seen for the bio oil HPUs (K3-4). The bio oil HPUs are rarely in use, and when in operation they are set at a static power level yielding poorer dynamic

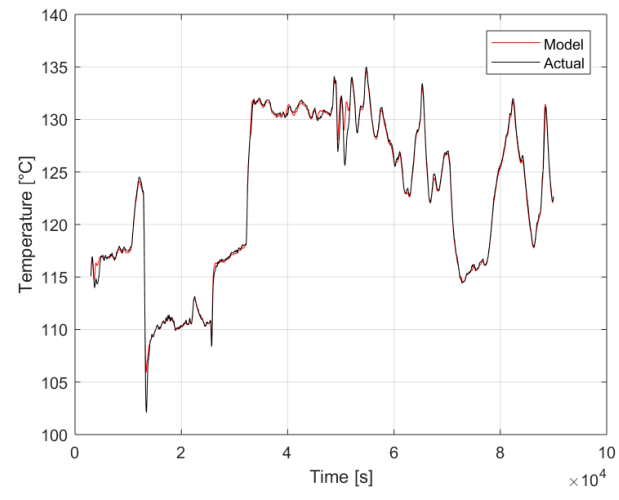

Figure 6. Plot of simulated response from identified model and actual data for $T_{k t}$. NRMSE: 0.932 .

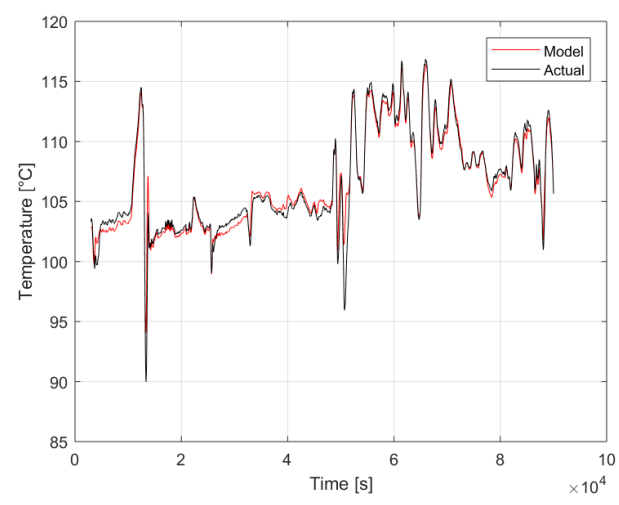

Figure 7. Plot of simulated response from identified model and actual data for $T_{n t}$. NRMSE: 0.787 .

Table 3. Parameters for process model matrix

\begin{tabular}{cc}
\hline Parameter & Transfer function \\
\hline$H_{11}(s)$ & $\frac{3.56}{1+122 s} e^{-5.2 s}$ \\
$H_{12}(s)$ & $\frac{0.996}{1+122 s} e^{-0.7 s}$ \\
$H_{21}(s)$ & $\frac{1.464}{(1+129 s)(1+0.017 s)} e^{-16.8 s}$ \\
$H_{22}(s)$ & $\frac{1.042}{1+93 s}$ \\
$H_{23}(s)$ & $\frac{0.360}{1+6.5 \cdot 10^{7} s} e^{-0.5 s}$ \\
$H_{24}(s)$ & $\frac{-0.046}{(1+22 s)(1+0.038 s)} e^{-24 s}$ \\
$H_{34}(s)$ & $\frac{1}{1+22.6 s}$ \\
\hline
\end{tabular}

quality of the estimation data. There might also be a larger nonlinear dynamic due to combustion process which our models does not represent properly. Further investigation into this should be done. 
Table 4. NRMSE fits for all identified HPU models.

\begin{tabular}{lcc}
\hline & \multicolumn{2}{c}{ NRMSE [\%] } \\
\cline { 2 - 3 }$H P U$ & $T_{k t}$ & $T_{n t}$ \\
\hline $\mathrm{K} 1$ & 93.2 & 78.7 \\
$\mathrm{~K} 2$ & 94.4 & 80.8 \\
$\mathrm{~K} 3$ & 64.4 & -9.6 \\
$\mathrm{~K} 4$ & 36.0 & 45.5 \\
\hline
\end{tabular}

\subsection{Model predictive control}

Figure 8 and 9 show the MPC performance compared to the existing PID cascade. The MPC use the identified model presented in section 7.3 as internal and external plant. The simulation use the historic $T_{n r}$ as MD. The historic $\dot{V}$ is set as $\operatorname{Re}_{\dot{V}}$. The simulation shows that MPC better able to keep $T_{n t}$ to the reference temperature. Figure 9 shows outputs and disturbances for HPU K1 in the upper section and controlled variables $\dot{V}$ and $\dot{Q}$ for HPU $\mathrm{K} 1$ in the bottom plot.
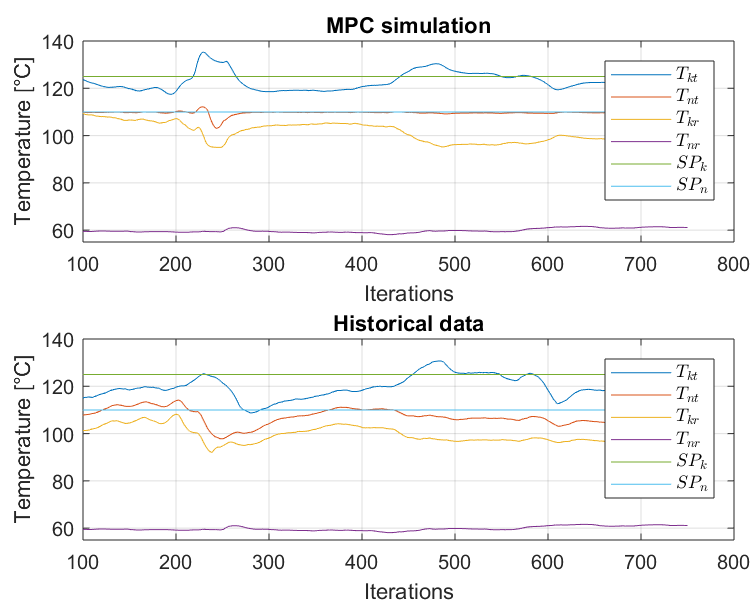

Figure 8. Comparison of closed loop control performance between MPC simulation and present PID cascade of K1 in respect to temperature.

\section{Discussion}

The load prediction algorithm worked quite well. Significant deviations from actual values are seen but averaged over longer periods the deviation is canceled. It is believed that a better prediction could be made if power used by consumers was used instead of power produced at the plants. Power production data may be influenced by the operators and may not correspond to actual consumer demand. There are also other methods using neural network that look promising, as shown by (Verrilli et al. 2017).

The unit commitment algorithm works well but may not be necessary for this system seeing as the HPUs have fast ramp rates and few restrictions. A combined optimization/MPC-scheme would likely be the best solu-
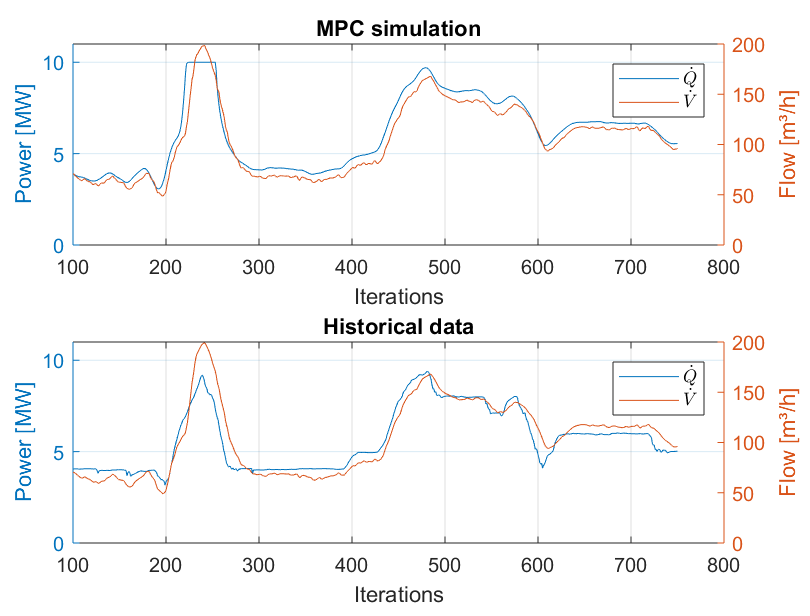

Figure 9. Comparison of closed loop control performance between MPC simulation and present PID cascade of K1 in respect to $\dot{Q}$ and $\dot{V}$.

tion, but would require writing a custom non-linear MPC algorithm.

Good correlation between model and plant was seen for the electrical HPUs. For the bio oil HPUs somewhat poor correlation was seen. This is mainly caused by lack of, and less dynamic estimation data which is detrimental for model estimation. There might also be differences in operation or some nonlinear dynamics in the combustion process which is not reflected in the estimation variables.

The MPC simulation shows that the temperature reference tracking may be improved by implementing MPC to control the HPU. However, the simulations was carried out using the identified models as internal and external plant, meaning there is $100 \%$ fit between the MPC's predicted response and the simulated system response. The MPC's actual performance is expected to be poorer.

Simulating the complete control hierarchy was difficult for a number of reasons. Currently the closed loop feedback used to indicate power demand is differential pressure across select customer heat exchangers. This is controlled by the customers and can therefore not easily be simulated without modeling the entire DHS. The relation between differential pressure and power demand is also not very clear. A simulation of the complete control hierarchy has therefore not yet been performed.

Further work is needed to complete the project, and our suggestions is to develop a nonlinear model of the HPUs and the DHS, which allows simulation of the control hierarchy and provides a more realistic MPC results.

\section{Conclusion}

Methods for load prediction and unit commitment performed quite well and are thought to be useful in a real implementation of the control hierarchy. The model predictive controller is likely to improve temperature reference tracking. The complete control hierarchy has not 
been tested and is necessary to complete the project.

\section{Acknowledgements}

The authors would like to thank Marius Hagalid for supplying the load prediction model and helping with our implementation. Karine Huuse, Ingeborg Sauge Torpe and Patrik Myrendal at Fortum Oslo Varme have aided our understanding of district heating systems in general and provided specific data necessary to complete the project.

\section{References}

Currie, Jonathan and David I. Wilson (2012). "OPTI: Lowering the Barrier Between Open Source Optimizers and the Industrial MATLAB User". In: Foundations of Computer-Aided Process Operations. Ed. by Nick Sahinidis and Jose Pinto. Savannah, Georgia, USA.

Fortum Oslo Varme (2018). Fjernvarme i Oslo. Retrieved 15.04.2018. URL: https: / / www . fortum . no / fjernvarme-i-oslo.

Gleixner, Ambros et al. (2017). The SCIP Optimization Suite 5.0. eng. Tech. rep. 17-61. Takustr.7, 14195 Berlin: ZIB.

Hagalid, Marius Jean Bischof (2018). "Development of Models for Automatic Control of the Facilities of Fortum Oslo Varme in Regard to Reliability and Optimal Operation". In: Unpublished preliminary report.

Lake, Andrew, Behnaz Rezaie, and Steven Beyerlein (2017). "Review of district heating and cooling systems for a sustainable future". In: Renewable and Sustainable Energy Reviews 67, pp. 417-425. ISSN: 13640321. DOI: https : / / doi .org/10.1016/j . rser.2016.09.061. URL: http:// www . sciencedirect . com / science / article / pii/S1364032116305585.

Löfberg, J. (2004). "YALMIP : A Toolbox for Modeling and Optimization in MATLAB". In: In Proceedings of the CACSD Conference. Taipei, Taiwan.

Lougee-Heimer, Robin (2003). "The Common Optimization INterface for Operations Research". English. In: IBM Journal of Research and Development 47(1), pp. 57-66.

Saravanan, B. et al. (2013). "A solution to the unit commitment problem-a review". In: Frontiers in Energy 7.2, pp. 223-236. ISSN: 2095-1698. DOI: 10.1007 / s11708-013-0240-3. URL: https : / / doi . org/10.1007/s11708-013-0240-3.

Statistisk Sentralbyrå (2017). Fjernvarme og fjernkjøling $i$ Norge. Retrieved 17.01.2018. URL: https: / / www . ssb.no/f jernvarme.

Verrilli, F. et al. (2017). "Model predictive control-based optimal operations of district heating system with thermal energy storage and flexible loads". English. In: IEEE Trans. Autom. Sci. Eng. (USA) 14.2, pp. 547-57. ISSN: 1545-5955. URL: http://dx.doi .org/10 . 1109/TASE.2016.2618948. 